\title{
Improved Efficiency Characterisation for Large Solid Angle SDD Detectors
}

\author{
P.J.Statham
}

Oxford Instruments Nanoanalysis, High Wycombe, Bucks HP12 3SE, U.K.

As EDX technology matures there is increasing expectation that accurate analysis can be achieved without standards. While standardless analysis with results normalised to $100 \%$ total is convenient, requires no expensive standards block and can work with conditions of unstable beam current, inaccuracies in fundamental parameters and detector efficiency are major sources of error. Furthermore, if some elements have been incorrectly identified or missed, normalised results for the correctly-identified elements can be seriously misleading. A calibration using standards for every element under a specific set of SEM conditions is required to perform accurate quantitative analysis in similar SEM configurations. If EDX spectrometer efficiency remains stable with time this calibration can be done once and for subsequent analyses at different beam currents only a single standard measurement is needed to determine the intensities for all other standards [1]. However, this calibration is expensive for the owner and it is desirable to have an accurate set of default standard measurements and an accurate efficiency characteristic for a particular detector type.

As reported last year [1], the efficiency of a small area INCAx-act SDD was determined by mounting the detector on the same test bed as a "gold standard reference" $\mathrm{Si}(\mathrm{Li})$ (6400GS) that had been calibrated using the Bessy II synchrotron [2]. Using relative measurements from the test bed and the original efficiency measurements made on 6400GS, an efficiency curve for INCAx-act was deduced and used to improve accuracy for single-standard quantitative analysis. For the next generation "X-Max" detector, the characterisation was expected to be much easier. Although X-Max uses a larger area SDD and a different manufacturing process, no low pressure nitrogen is used for encapsulation so the efficiency characteristic is simpler than the $\mathrm{x}$-act. A first attempt was made using a standard SEM with nominal $30^{\circ} \mathrm{TOA}$, rather than the $35^{\circ} \mathrm{TOA}$ test bed. A different block of standards was used and no special precautions were taken to ensure accurate $\mathrm{kV}$ and geometry. Rather than bother mounting the 6400GS on the same microscope, theoretical corrections were used to convert the $35^{\circ}$ test bed results to what would be expected at $30^{\circ}$. However, the results were disappointing showing a high degree of scatter and some points that could not be explained by any parameterised efficiency curve. In particular, the efficiency at $\mathrm{C}$ was substantially lower than any reasonable curve would predict. Possible reasons for the discrepancies were investigated.

The two different standard blocks both had a purportedly "reliable" vitreous carbon standard. However by comparison on the same instrument, it was found that the vitreous carbon standard used for the new experiments emitted $16 \%$ less intensity than the standard used for the $6400 \mathrm{GS}$ measurements! Another suspected source of error was the range of TOA subtended by the large area detector. Although absorption is almost linear with TOA for heavy absorption, for intermediate absorption there is non linearity and the average intensity detected is slightly lower than the intensity obtained at the average TOA. Modelling exposed the worst condition for non-linearity (absorption factor approximately 0.44 ) and for x-rays from a range of $25^{\circ}$ to $45^{\circ}$, the average intensity was only $0.7 \%$ less than that at the $35^{\circ}$ average TOA. Although this geometry issue was not a significant source of error, variable small tilts of individual standards on the standards block were found to 
produce changes in intensity, particularly at energies below $1 \mathrm{keV}$. A further issue with a large area detector is the ribbed support for the window foil. Absorption effects are rather complicated at high solid angles because of the transmission of x-rays through the "corners" of the ribs. A simulation model was set up to calculate the total transmission for various situations and some results are shown in fig. 1 for a $50 \mathrm{~mm}^{2}$ detector at various specimen-detector distances.

Prepared with this new information, characterisation was repeated with the following precautions:

1) Same (LEO 1450) microscope with $35^{\circ}$ TOA used for both 6400GS and X-Max detectors

2) Special tilt stage used to check TOA is correct after each detector installation

3) Same physical standards used for the comparisons, in particular the vitreous carbon

4) Measurements taken with zero and $180^{\circ}$ rotation of the stage and averaged in order to cancel out effects due to variable tilt of individual standards

5) Distance of specimen to detector determined to calculate window ribs absorption characteristic. This second experiment produced much less scatter in results. However, a simple parameterisation, using Heinrich absorption coefficients and expected detector parameters, produced a relative efficiency curve that was a very poor approximation below $1 \mathrm{keV}$ (see dashed red curve in Fig.2).

Therefore, rather than using a simple parameterised fit, Bessy II measurements of a real window ([2],[3]) were used to deduce the foil transmission, excluding the effect of the ribs. This foil transmission was used to account for changes in polymer thickness, rib transmission was calculated with the new model and CXRO-parameterised absorption curves were used to correct for changes in thickness of other layers. Fig.2 shows the improved fit to the relative efficiency measurements and the X-Max efficiency deduced from that for 6400GS. The improved efficiency file for X-Max now enables single-standard quantitative results to be obtained with previously-reported accuracy [1] and the improved characterisation at low energy is a key pre-requisite for nano-scale analysis at low $\mathrm{kV}$.

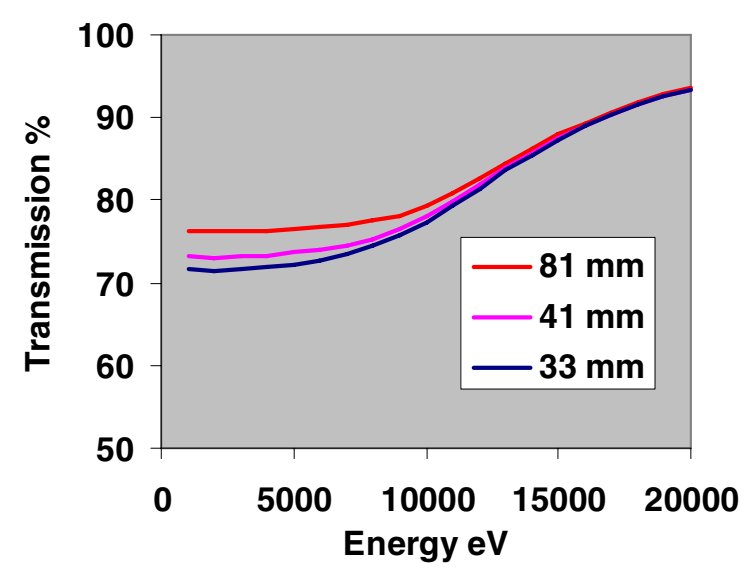

Fig. 1. Transmission of $50 \mathrm{~mm}^{2}$ silicon rib support at 33,41 and $81 \mathrm{~mm}$ distance from point source.

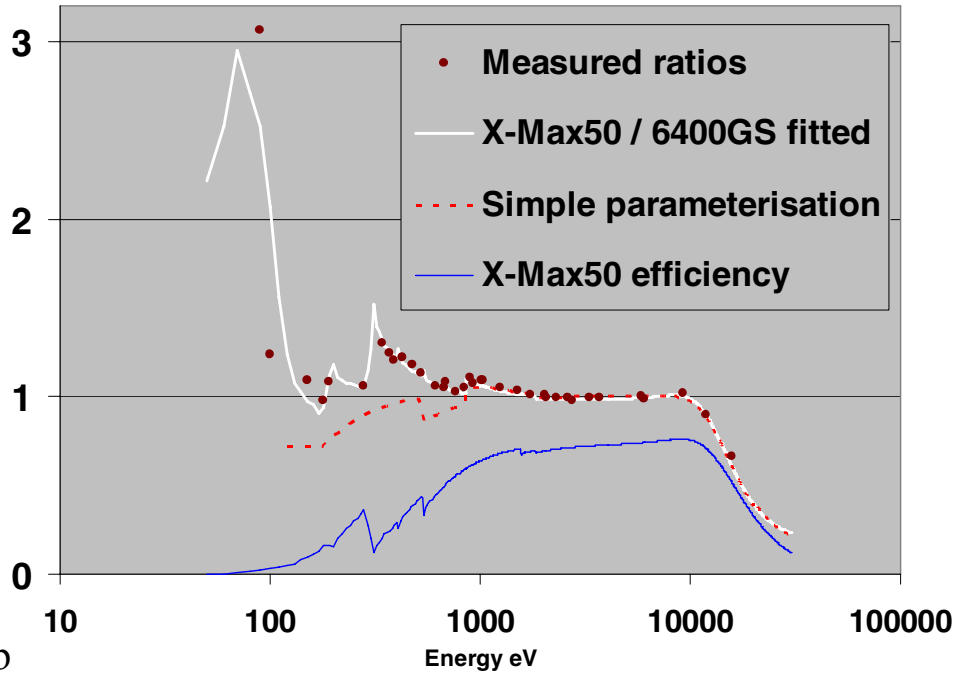

Fig.2. Measured and fitted (white) efficiency ratios for X-Max $50 \mathrm{~mm}^{2}$ detector relative to 6400GS Si(Li). Deduced X-Max curve below.

References

[1] P. Statham, Microsc Microanal 15(Suppl 2), 528, 2009

[2] M. Alvisi. et al.. Microsc. Microanal. 12, 406-415, (2006)

[3] F. Scholze and M. Procop, X-Ray Spectrom. 34, 473-476, (2005) 